Verhaltenstherapie 1997;7:201-203

\title{
Contents, Vol. 7, No. 4, 1997
}

Verhaltenstherapie

Vol. 7, Heft 4, Dezember 1997

Vol. 7, Heft 4, December 1997

Originalarbeiten

Original Papers

204 Verhaltenstherapie in der Klinischen Neuropsychologie

Clinical Neuropsychology (2):

(2): Verhaltensanalyse und Verhaltensmodifikation Behavior Analysis and Behavior

Modification of a

eines Patienten mit traumatisch erworbenem Patient with a Traumatically Acquired Frontal Lobe Frontalhirn-Syndrom Syndrome

Heubrock, D.; Peternann. F. (Bremen) Heubrock, D.; Petermann, F. (Bremen)

217 Beziehungen zwischen Parametern der impliziten und expliziten Informationsverarbeitung und psychosomatischen Selbstberichtsmaßen

Scholz, O.B.; Ott, R.; Müller-Sinik, K. (Bonn)

217 Relation between Parameters of Implicit and

Explicit Cognition and Psychosomatic Self-Report Measures

Scholz, O.B.; Ott, R.; Müller-Sinik, K. (Bonn)

Aus der Praxis für die Praxis

For the Practitioner

226 Biographisch individualisierte Planung und

Durchführung von Verhaltenstherapien - Hinweise und Erfahrungen aus der Praxis

Zarbock, G. (Hamburg)

226 Biographically Individualized Treatment Plans and Application of Behavior Therapy - A Contribution from a Practitioner

Zarbock, G. (Hamburg)

Falldarstellung

Case Report

235 Synergetische Wirkung zwischen Verhaltenstherapie 235 Synergistic Effects between

Behaviour Therapy and

und SSRI-Behandlung? Falldarstellung einer Patientin SSRI Treatment? A Case Report of a Patient with

mit therapierefraktärer Depression Treatment-Resistant Depression

Peter, H; Stark, F.-M.; Lauterbach, B.; Naber, D. (Hamburg) Peter, H.; Stark, F.-M.;

Lauterbach, B.; Naber, D. (Hamburg) 
243 Neuigkeiten vom Buch- und Zeitschriftenmarkt

243 Articles and Books

248 Mitteilungen der Verbände

248 Arbeitsgemeinschaft für Verhaltensmodifikation e.V. (AVM-D)

Berufsverband Deutscher Psychologinnen und Psychologen e. V. (BDP)

Deutsche Gesellschafl für Verhaltenstherapie e.V. (DGVT)

1FT Institut für Therapieforschung

Institut für Verhaltenstherapie e. V., Berlin

248 Information by Behavior Therapy Associations

256 Tagungen und Kongresse

256 Meetings and Conferences

215 Erratum

215 Erratum

Autorenverzeichnis 1997

Sachwortverzeichnis 1997

III Jahres-Inhalt 1997 (nach Seite 262)

259 Author Index 1997261 Subject Index 1997 IV Complete Contents 1997 (following page 262)

202 Impressum

V Hinweise für Autoren

202 Imprint

VI Instructions to Authors

KAHGER

(C) 1997 S. Karger GmbH, Freiburg Fax (07 61)45207 14 http://www.karger.ch 203 